\title{
The lag time from symptoms onset of rheumatoid arthritis to initiation of Methotrexate: Data from the Moroccan register of biotherapies
}

Eddaoudi $\mathrm{M}^{1 *}$, Rostom $\mathrm{S}^{1}$, Hmamouchi ${ }^{2,3}$, El Binoune $\mathrm{I}^{1}$, Amine $\mathrm{B}^{1}$, Abouqal R ${ }^{2}$, Achemlal L ${ }^{4}$, Allali $\mathrm{F}^{5}$, El Bouchti I ${ }^{6}$, El Maghraoui $\mathrm{A}^{7}$, Ghozlani $I^{8}$, Hassikou $\mathrm{H}^{9}$, Harzy $\mathrm{T}^{10}$, Ichchou $\mathrm{L}^{11}$, Mkinsi $\mathrm{O}^{12}$, Niamane $\mathrm{R}^{13}$, and Bahiri $\mathrm{R}^{1}$

${ }^{1}$ Department of Rheumatology, A, El Ayachi Hospital, Ibn Sina University Hospital, Rabat-Sale, Morocco

${ }^{2}$ Laboratory of Epidemiology and Clinical Research, Faculty of Medicine and Pharmacy, Rabat, Morocco

${ }^{3}$ Provincial Hospital of Skhirat-Temara, Morocco

${ }^{4}$ Department of Rheumatology, Military Hospital Mohamed V, Ibn Sina University Hospital, Rabat, Morocco

${ }^{5}$ Department of Rheumatology B, El Ayachi Hospital, Ibn Sina University Hospital, Rabat-Salé, Morocco

${ }^{6}$ Department of Rheumatology, Arrazi Hospital, University Hospital Mohammed VI, Marrakech, Morocco

${ }^{7}$ Rheumatology medical office, Rabat, Morocco

${ }^{8}$ Department of Rheumatology, University Hospital Agadir, Morocco

${ }^{9}$ Department of Rheumatology, Military Hospital Meknes, Hassan II University Hospital, Morocco

${ }^{10}$ Department of Rheumatology, University Hospital Hassan II Fès, Morocco

${ }^{11}$ Department of Rheumatology, University Hospital Mohammed VI Oujda, Morocco

${ }^{12}$ Department of Rheumatology, University Hospital Ibn Rochd, Casablanca, Morocco

${ }^{13}$ Department of Rheumatology, Military Hospital Avicenne, University Hospital Mohammed VI, Marrakech, Morocco

\begin{abstract}
Objective: The purpose of this study is to determine the time between the onset of rheumatoid arthritis (RA) symptoms and the introduction of methotrexate, as well as the associated factors.

Methods: A multicenter cross-sectional study that included data from the Moroccan register of biologic patients with rheumatoid arthritis. Sociodemographic, economic patient data and disease data were collected. A logistic regression was performed to determine the factors that are associated with the change in the delay between the onset of symptoms and the initiation of cDMARD.

Results: A total of 225 RA patients were included. The mean age was $51 \pm 11$ years, with a female predominance of $88 \%$ ( $\mathrm{n}=197$ ). The diagnosis lag time was 12 months (12-48). Seventy-three percent of the cases were housewives. Seventy-nine percent of patients received Methotrexate as the first CDMARDS. Salazopyrine was the second prescribed CDMARDS with 7\%. Hydroxychloroquine was the third prescribed cDMARDs which $2.2 \%$. The median lag time from symptoms onset to initiation of Methotrexate was 24 months (0.16-71). The factors, associated with the change in the delay between the onset of symptoms and the introduction of methotrexate in univariate analysis, were delay in diagnosis and corticosteroids Taking $(\mathrm{p}<0.05)$, only the delay of diagnosis in multivariate analysis persists $\mathrm{p}=0.0001$.

Conclusions: Our study suggests that there is a significant delay between diagnosis and the introduction of methotrexate as a first line cDMARDs. In multivariate analysis only the time to diagnosis was associated with the time to start methotrexate.
\end{abstract}

\section{Introduction}

Rheumatoid arthritis (RA) is a chronic autoimmune inflammatory disease that affects large and small joints causing bone erosions, joint deformities, and comorbidities [1-3].

Early initiation of the Conventional Disease Modifying AntiRheumatic Drugs (cDMARDs) within 2 months from the onset of symptoms provides better control over the disease activity and prevents irreversible cartilage damage in the long term [1,4-6]. Conversely, the increase of the lag time between the onset of symptoms and the introduction of cDMARDs is associated with a risk of joint destruction and permanent disability [1].
Guidelines recommend the early referral of the general practitioner to the rheumatologist in order to confirm the diagnosis of RA and the early initiation of treatment $[7,8]$, and also to ensure the monitoring and evaluation of patients and the response to treatment [9].

*Correspondence to: Meryem Eddaoudi, Department of Rheumatology, A, El Ayachi Hospital, Ibn Sina University Hospital, Rabat-Sale, Morocco, E-mail mery.eddaoudi@gmail.com

Key words: delay, lag time, methotrexate, rheumatoid arthritis, symptom onset

Received: January 10, 2021; Accepted: Janauary 22, 2021; Published: Janauary 29, 2021 
The literature review shows that there are several factors associated with the initiation delay of cDMARDs [5]. In the Moroccan context, few data are available about the factors that influence the instauration delay of cDMARDs.

The aim of our study was to assess the lag time from symptoms onset to initiation of conventional DMARDs and to explore the factors associated with lag time in a sample of Moroccan patients.

\section{Methods}

\section{Study design}

The details of the data collected have been published previously [10]. We included 225 patients followed for rheumatoid arthritis treated by bDMARDs in different university hospitals in Morocco and meeting the criteria ACR-EULAR 2010.

Demographic data related to the patients included age, sex, educational level, type of health assurance and date of symptom onset reported by the patient or from patient chart.

The clinical and biological data collected were sedimentation rate (ESR), protein-C-reactive (CRP), the Disease Activity Score (DAS28ESR), rheumatoid factor (RF) and anti-citrullinated peptide antibody (ACPA) status, erosion presence.

We calculated the lag time from the symptom's onset of RA to the initiation of cDMARDs.

The factors considered in analysis included: age, sex, anti-CCP and RF positivity, serum ESR and CRP, DAS28, taking analgesics and taking corticosteroid, erosion presence and diagnosis delay.

\section{Statistical analysis}

Descriptive statistics were presented as mean and standard deviation (SD) or as median and inter quartile range (IQR) according to the distribution, and qualitative variables were presented as a frequency and percentage.

We chose as to cut off the lag time initiation of Methotrexate 24 months (Methotrexate was chosen because it is the most prescribed treatment as the first cDMARDs).

We performed a univariate analysis using a comparison test, and multivariate analysis to determine the factors associated with the increase of the lag time of methotrexate initiation.

\section{Results}

A total of 225 patients with rheumatoid arthritis were included from the RBSMR data. The mean age of patients was $52 \pm 11.36$ years. The majority of patients were females $\mathrm{N}=197$ (87.60\%). Ninety percent of patients were ACPA positive and displayed the rheumatoid factor (Table 1).

The first cDMARD that was initially used was methotrexate (79\%), followed by salazopyrin $7 \%$ and leflunomide (1.3\%) and others $(12.7 \%)$. The mean of disease duration was $14.14 \pm 9.05$ years. The median of diagnosis lag time was 12 months (12-48) (Table 2).

The lag time between the symptom's onset and the initiation of methotrexate was 24 months (0.16-71) and the lag time in initiating Salazopyrin was 36 months (14-95) (Table 2).

In univariate analysis, we found in the group, which had the lag time of Methotrexate initiation more than 24 months, a longer
Table 1. Demographics and clinical features of the patient sample

\begin{tabular}{|c|c|}
\hline Features & $N$ : 225 \\
\hline Age $^{1}$ (years) & $51 \pm 11,36$ \\
\hline Female sex ${ }^{2}$ & $197(87,60)$ \\
\hline \multicolumn{2}{|l|}{ Marital status } \\
\hline - Married $^{2}$ & $162(72)$ \\
\hline - Single ${ }^{2}$ & $28(12,4)$ \\
\hline - Widowed ${ }^{2}$ & $18(8)$ \\
\hline - Divorced & $16(7,1)$ \\
\hline \multicolumn{2}{|l|}{ Level of education } \\
\hline - Illiterate $^{2}$ & $99(44)$ \\
\hline - Primary studies ${ }^{2}$ & $42(18,7)$ \\
\hline - Secondary studies ${ }^{2}$ & $52(23,1)$ \\
\hline - Higher education ${ }^{2}$ & $22(9,8)$ \\
\hline Sedimentation rate ${ }^{1}\left(\mathrm{~mm} / 1^{\text {st }}\right.$ hour $)$ & $42 \pm 24$ \\
\hline C-reactive protein ${ }^{3}(\mathrm{mg} / \mathrm{l})$ & $22(8-41,5)$ \\
\hline $\mathrm{DAS}^{2} 8^{1}$ & $5,2 \pm 1$ \\
\hline Positive rheumatoid factor*2 & $192(90,5)$ \\
\hline Positive Anti-ccp*2 & $151(88,8)$ \\
\hline
\end{tabular}

1: Mean and standard deviation; 2: Number and percentage; 3: Inter-quartile median; * Data available

Table 2. The first cDMARDs initiation and the lag time of Methotrexate initiation

\begin{tabular}{|c|c|}
\hline Features & $N: 225$ \\
\hline \multicolumn{2}{|l|}{ First cDMARDs: } \\
\hline Methotrexate $^{2}$ & 177 (79) \\
\hline Salazopyrine $^{2}$ & $15(7)$ \\
\hline Leflunomide $^{2}$ & $3(1,3)$ \\
\hline Other $^{2}$ & $30(12,7)$ \\
\hline Initiation Methotrexate lag time ${ }^{3}$ (months) & $24(0.16-71)$ \\
\hline Duration of evolution $^{1}$ (years) & $14,14 \pm 9,05$ \\
\hline Diagnosis lag time ${ }^{3}$ (months) & $12(12-48)$ \\
\hline
\end{tabular}

1: Mean and standard deviation; 2: Number and percentage; 3 : Inter-quartile median

diagnostic delay ( $\mathrm{p}=0.0001$ ). The use of corticosteroids was important in the group that had the methotrexate initiation lag time $\leq 24$ months $(\mathrm{p}=0.02)$. On the other hand, no difference in age, sex, seropositivity, erosive form of rheumatoid arthritis and analgesic intake was found. In multivariate analysis, only the diagnostic lag time was associated with the initiation delay of Methotrexate $>24$ months (Table 3).

\section{Discussion}

This study suggested that there is a delay between onset of symptoms and diagnosis, and initiation of cDMARDS treatment in RA patients. The median diagnostic lag time was 12 months (12-48) and the median lag time between initial presentation of RA and methotrexate instauration was 24 months (0.16-71).

More than $75 \%$ of patients with rheumatoid arthritis have erosions in the first two years of illness $[11,4]$. The current consensus is the need for early diagnosis and rapid initiation of treatment with cDMARDs to prevent destruction and joint damage [4,12-16].

In an American study by Kimsey and al [1], the time between symptoms onset and initiation of cDMARDs was 4 months (SD 5.8), and in an Australian study was 6,6 months (18-52.1) [2].

In the Danish nationwide DANBIO registry including 10416 patients with rheumatoid arthritis, they studied the changes of the diagnosis delay from year 2000 to 2011. The mean duration from initial symptoms to diagnosis for RA declined from 29 months (year 2000) to 3-4 months (year 2011) [14]. 
Table 3. Factors Associated with the variation in the Time of Methotrexate initiation in RA

\begin{tabular}{|c|c|c|c|c|c|c|}
\hline \multirow[b]{2}{*}{ Features } & \multicolumn{3}{|c|}{ Univariate analysis } & \multicolumn{3}{|c|}{ Multivariate analysis } \\
\hline & $\begin{array}{c}\text { Methotrexate } \\
\text { initiation time }>\mathbf{2 4} \\
n=64 / 130\end{array}$ & $\begin{array}{c}\text { Methotrexate } \\
\text { initiation time } \leq \mathbf{2 4} \\
n=66 / 130\end{array}$ & $\mathbf{p}$ & OR & IC & $\mathbf{p}$ \\
\hline Age $^{1}$ (years) & $52 \pm 11$ & $52 \pm 11$ & 0,6 & - & - & - \\
\hline Female sex ${ }^{2}$ & $58(90,6)$ & $56(84,8)$ & 0,3 & - & - & - \\
\hline Sedimentation rate $^{1}$ (mm / 1st hour) & $39,7 \pm 25$ & $43,5 \pm 21$ & 0,3 & - & - & - \\
\hline C-reactive protein ${ }^{3}(\mathrm{mg} / \mathrm{l})$ & $15(6-35)$ & $27(9-47)$ & 0,2 & - & - & - \\
\hline DAS28 ${ }^{1}$ & $4,9 \pm 1,1$ & $5,4 \pm 0,9$ & 0,05 & 0,7 & {$[0,47-1,28)$} & 0,3 \\
\hline Diagnosis lag time ${ }^{3}$ (months) & $48(12-72)$ & $5(1-12)$ & 0,0001 & 1,06 & {$[1,03-1,09)$} & 0,0001 \\
\hline Positive rheumatoid factor ${ }^{2}$ & $58(92,5)$ & $54(91,5)$ & 0,9 & - & - & - \\
\hline Positive Anti-ccp ${ }^{2}$ & $36(81,8)$ & $54(93,1)$ & 0,08 & 0,7 & {$[0,46-1,32)$} & 0,3 \\
\hline Carpite / Erosions ${ }^{1}$ & $30(46,9)$ & $27(40,9)$ & 0,5 & - & - & - \\
\hline Taking corticosteroids ${ }^{1}$ & $59(92,2)$ & $66(100)$ & 0,02 & - & - & - \\
\hline Taking analgesic ${ }^{1}$ & $31(48,4)$ & $34(51,5)$ & 0,9 & - & - & - \\
\hline
\end{tabular}

1: Mean and standard deviation; 2: Number and percentage; 3: inter-quartile median

In a French study, Fautrel et al. [17], including 813 patients from a national claims database, the lag time from the symptoms onset and the first physician visit (lag1) was 0.5 months and the time between initial visit to rheumatology referral (lag2) was 1 month. In a Spanish cohort, the median lag time between symptom onset and the first visit to a rheumatologist was 17 months and between onset of symptoms and initiation of treatment by DMARD was 19 months [18].

In the ESPOIR cohort, the lag time before referral was $103.1 \pm 52.4$ days [19]. In a study conducted in 10 centers in Europe, the median time between onset of symptoms and diagnosis by the rheumatologist ranges from 12 to 24 weeks [20].

In the study by Massardo et al. [21] in Chilean patients, the lag time between symptoms and initial consultation ranged from 3 to 6 years.

In the study of Polanco et al. [4] based on the Venezuelan population, the diagnostic lag time was 40.5 months (1-424). Riad M et al. [22] included 152 RA patients, 35\% were Caucasian, 37\% Black, $20 \%$ Hispanic and $8 \%$ others. The median time to first rheumatology visit was 22.7 months in the group Spanish and 6-8 months for other groups.

Our study has demonstrated that the lag time between the onset of symptoms and the initiation of Methotrexate was longer in comparison with other studies conducted in some developed countries (United States of America, France and Denmark).

Furthermore, other studies realized at the level of some middle- or low-income countries (Chile and Venezuela) have shown a relatively long delay compared to that observed by our study conducted in Morocco.

In our study, the factors associated with the change in Methotrexate initiation lag time were the diagnostic delay and corticosteroids taking. Patients who started Methotrexate after 24 months had a higher diagnosis delay, while taking corticosteroids was greater in the group of patients who received Methotrexate before 24 months.

On the other hand, we did not find any difference concerning the age, sex, the seropositivity, the erosive form and the taking of analgesic.

In multivariate analysis, only the diagnosis delay is identified as a factor associated with the Methotrexate initiation delay.

Our results are similar to those of a Spanish study [18] where the variable that is associated with a delay of initiation of treatment was the lag time from the onset of symptom to the first rheumatologist consultation. Furthermore, this study had also mentioned the level of education as a factor associated with the DMARDs therapy delay.

In the study of Chan et al. [23], the factors associated with shorter delays were disease progression and rheumatoid factor seropositivity, but no correlation was found with age, sex, or co-morbidities.

In Kimsey et al. [1], opioids and no steroidal anti-inflammatory drugs were the factors associated with delayed initiation of cDMARDs treatment.

Polanco et al. [4] found that the first consultation with a general practitioner at a public health center as well as with an orthopedic surgeon were the main factors associated with the increasing of the delay of the diagnosis and the introduction of cDMARDs. In agreement with the results of Matthew et al. [2] who found that the factors that are associated with a delay in cDMARDs introduction were the presentation of the patient to the general practitioner, the delay of referral from the general practitioner to the rheumatologist as well as the socio-economic disadvantage, fatigue score and low DAS28.

In fact, the delay of referral from the general practitioner to the rheumatologist is an important factor influencing the delay of diagnosis. Studies have shown that there is poor agreement in the diagnosis of RA between rheumatologists and general practitioners [4,24,25].

The limitations of our study are its retrospective nature, the collection of the database from the medical records and therefore missing data like the referral lag time to the rheumatologist. Secondly, the recall bias may occur during the patient interrogatory regarding the date of symptoms onset which is based on the memory of the patient. But this study presents strong points. It is a multicentric study of the first Moroccan and African register of biotherapies.

In order to ensure an early diagnostic and an early treatment, it is necessary to sensitize general practitioners to refer patients to a rheumatologist and raise the awareness of patients who medicate themselves with corticosteroids.

\section{Conclusions}

In conclusion, this study, which was based on data from the Moroccan register of biotherapy patients with RA, suggests that there is a significant delay from the diagnosis to the initiation of treatment with cDMARDs. The delay of diagnosis was the factor associated with the Methotrexate initiation delay. Further large-scale studies are needed to confirm these results. 


\section{Declarations}

Ethics approval and consent to participate: The protocol for the original RBSMR study was reviewed and approved by local institutional review boards and the national ethic committee: Ethics committee for biomedical research Mohammed V university- RABAT.

Faculty of medicine and pharmacy of RABAT. The committee's reference number: 117/17

Consent to publish: This project has been reviewed and accepted by the scientific committee of the RBSMR study. Moreover, this committee has reviewed this current manuscript and has agreed upon its submission to your journal.

Availability of data and materials: All data generated or analyzed during this study are included in this published article.

Acknowledgements: The authors would like to thank the scientific Committee and national principal investigators of the RBSMR study: Lahcen Achemlal, Fadoua Allali, Rachid Bahiri, Imane El Bouchti, Imad El Ghozlani, Abellah El Maghraoui, Toufik Harzy, Ihsane Hmamouchi, Linda Ichchou, Ouafa Mkinsi, and Redouane Niamane; patients who agreed to participate in this study.

Funding information: Data collection for the Moroccan Registry of biotherapy "Registre de Biothérapies de la Société Marocaine de Rhumatologie" (RBSMR) was supported by an unrestricted grant from Pfizer, Novartis, Janssen, and Abbvie. The ancillary study described in this manuscript was conducted without any type of funding.

\section{Competing interests: No competing interests}

Authors' Contributions: We declare that we participated at the study as following:

ME performed the statistical analysis and interpretation and prepared the manuscript. SR participated in article writing and critical review of the manuscript. IH reviewed and interpreted the statistical analysis. RB participated in critical review of the manuscript. All authors read and approved the final manuscript.

\section{References}

1. Kimsey L, Weissman JS, Patel A, Drew A, Koehlmoos T, et al. (2019) Delay in Initiation of DMARD or Anti-Inflammatory Therapy in Patients Newly Diagnosed with Rheumatoid Arthritis: An Analysis of United States Military Health System TRICARE Beneficiaries. Semin Arthritis Rheum 48: 821-827. [Crossref]

2. Ison M, Duggan E, Mehdi A, Ranjeny T, Benham H (2018) Treatment delays for patients with new onset rheumatoid arthritis presenting to an Australian early arthritis clinic. Intern Med J 48: 1498-1504. [Crossref]

3. Pincus T, Callahan LF (1989) Reassessment of twelve traditional paradigms concerning the diagnosis, prevalence, morbidity and mortality of rheumatoid arthritis. Scand $J$ Rheumatol 79: 67-96. [Crossref]

4. Rodriguez-Polanco E, Al Snih S, Kuo Y-F, Millan A, A. Rodriguez M (2011) Lag time between onset of symptoms and diagnosis in Venezuelan patients with rheumatoid arthritis. Rheumatol Int 31: 657-665. [Crossref]

5. Barhamain AS, Magliah RF, Shaheen MH, Munassar SF, Falemban AM, et al. (2017) The journey of rheumatoid arthritis patients: A review of reported lag times from the onset of symptoms. Open Access Rheumatology 9: 139-150. [Crossref]
6. Harris ED Jr. (1990) Rheumatoid arthritis: pathophysiology and implications for therapy. N Engl J Med 322: 1277-1289. [Crossref]

7. Emery P, Breedveld FC, Dougados M, Kalden JR, Schiff MH, et al. (2002) Early referral recommendation for newly diagnosed rheumatoid arthritis: evidence-based development of a clinical guide. Ann Rheum Dis 61: 290-297. [Crossref]

8. Lacaille D, Aslam HA, Daphne PG, Esdaile JM (2005) Gaps in Care for Rheumatoid Arthritis: A Population Study. Arthritis Rheum 53: 241-248. [Crossref]

9. American College of Rheumatology Subcommittee on Rheumatoid Arthritis Guidelines (2002) Guidelines for the management of rheumatoid arthritis: 2002 Update. Arthritis Rheum 46: 328-346. [Crossref]

10. Hmamouchi I, Abouqal R, Achemlal L, Allali F, Bahiri R, et al. (2019) The Moroccan registry of biological therapies in rheumatic diseases (RBSMR): methods and preliminaries results. Rev Mar Rhum 49: 32-37.

11. Van der Heidi A, Jacobs JWG, Bijlsma JWJ, A H Heurkens, C van Booma-Frankfort, et al. (1996) The Effectiveness of Early Treatment With "Second-Line" Antirheumatic Drugs. A Randomized, Controlled Trial. Ann Intern Med 124: 699-707. [Crossref]

12. Boers M (2003) Understanding the window of opportunity concept in early rheumatoid arthritis. Arthritis Rheum 48: 1771-1774. [Crossref]

13. Aletaha D, Eberl G, Nell V, Machold K, Smolen J (2002) Practical progress in realisation of early diagnosis and treatment of patients with suspected rheumatoid arthritis: results from two matched questionnaires within three years. Ann Rheum Dis 61: 630-634. [Crossref]

14. Sorensen J, Hetland ML (2015) Diagnostic delay in patients with rheumatoid arthritis, psoriatic arthritis and ankylosing spondylitis: results from the Danish nationwide DANBIO registry. Ann Rheum Dis 74: e12. [Crossref]

15. Bykerk V, Emery P (2010) Delay in receiving rheumatology care leads to long-term harm. Arthritis Rheum 62: 3519-3521. [Crossref]

16. Lard LR, Visser H, Speyer I, vander Horst-Bruinsma IE, Zwinderman AH, et al. (2001) Early versus delayed treatment in patients with recent-onset rheumatoid arthritis: comparison of two cohorts who received different treatment strategies. Am J Med 111: 446-451. [Crossref]

17. Fautrel B, Joubert J-M, Cukierman G, Laurendeau C, Gourmelen J, et al. (2013) THU0512 Rheumatoid arthritis (RA), comorbidities and biological agents' uptake in France: analysis of a national claims database. Ann Rheum Dis 72: A337.

18. Hernandez-Garcia C, Vargas E, Abasolo L, Abásolo L, Lajas C, et al. (2000) Lag time between onset of symptoms and access to rheumatology care and DMARD therapy in a cohort of patients with rheumatoid arthritis. J Rheumatol 27: 2323-2328. [Crossref]

19. Combe B, Rincheval N, Benessiano J, Berenbaum F, Cantagrel A, et al. (2013) Fiveyear favorable outcome of patients with early rheumatoid arthritis in the 2000s: data from the ESPOIR cohort. J Rheumatol 40: 1650-1657. [Crossref]

20. Raza K, Stack R, Kumar K, Filer A, Detert J, et al. (2011) Delays in assessment of patients with rheumatoid arthritis: variations across Europe. Ann Rheum Dis 70: 18221825. [Crossref]

21. Massardo L, Aguirre V, García ME, Cervilla V, Nicovani S, et al. (1995) Clinical expression of rheumatoid arthritis in Chilean patients. Semin Arthritis Rheum 25: 203213. [Crossref]

22. Riad M, Dunham DP, Chua JR, Shakoor N, Sobia H, et al. (2020) Health Disparities Among Hispanics With Rheumatoid Arthritis: Delay in Presentation to Rheumatologists Contributes to Later Diagnosis and Treatment. J Clin Rheumatol 26: 279-284. [Crossref]

23. Chan KA, Nelson DT, Walter AM (1994) The lag time between onset of symptoms and diagnosis of rheumatoid arthritis. Arthritis Rheum 37: 814-820. [Crossref]

24. Friedman DX, Flores MV, De la Rosa CH (2006) Criteria used by primary care physicians for the diagnosis and the referral to a rheumatologist of patients with rheumatoid arthritis. Reumatol Clin 2: 235-238.

25. Criswell LA, Such CL, Yelin EH (1997) Differences in the Use of Second-Line Agents and Prednisone for Treatment of Rheumatoid Arthritis by Rheumatologists and NonRheumatologists. J Rheumatol 24: 2283-2290.

Copyright: (C2021 Eddaoudi M. This is an open-access article distributed under the terms of the Creative Commons Attribution License, which permits unrestricted use, distribution, and reproduction in any medium, provided the original author and source are credited. 